\title{
Mobile Application Development for Optimal and Rapid Diagnosis of Vine Diseases
}

\author{
Liliana TOMOIAGA*, Cristian TOMOIAGA, Claudia TODORAN \\ Research Station for Viticulture and Enology Blaj, Romania \\ *)Corresponding author, e-mail: tomoiagaliliana@yahoo.com \\ BulletinUASVM Horticulture 74(1) / 2017 \\ Print ISSN 1843-5254, Electronic ISSN 1843-5394 \\ DOI:10.15835/buasvmcn-hort:12228
}

\begin{abstract}
In the vine culture late and inaccurate identification of the damage caused by diseases and pests can cause significant crop losses or even the loss of the entire production. Between 2010 and 2014, thousands of growers have accessed the online version of the guide requesting information, often too late to act effectively. Thus finding a new solution, easily accessible to growers that would offer a faster diagnosis, was necessary.
\end{abstract}

Keywords: agro-ecological infrastructure, biodiversity, diagnosis

\section{INTRODUCTION}

In a perennial culture such as the vine, late and inaccurate identification of the damage created by diseases and pests can cause significant crop loss or even the loss of the entire production. To support the growers in Romania, in 2010, it was printed and also posted online on https:// www.scvblaj.ro a guide on how to diagnose vine diseases and pests that describes in detail the most important pathogens together with pictures and various solutions in order to help the growers to bring the plants back to a healthy state whenever possible. Given the high number of growers in our country, especially small family growers, the guide is addressed to anyone, regardless of their past experience.

Between 2010 and 2014, thousands of growers have accessed the online version of the guide and contacted us to request additional help. It was gathered a lot of valuable information during that time but most notably the fact that many growers were contacting us too late, when a disease had already spread beyond any hope of recovery or worse after a wrong treatment was applied because of an inaccurate diagnosis.
In recent years we noticed an increasing number of wrong diagnostics of vine diseases and a tendency for pathogens to be more aggressive. In this time is necessary to find and put into practice new solution, easily accessible to growers that would offer a faster, easier and diagnosis.

Recent developments in the field of machine learning (Ghahramani, 2015), breakthroughs in computer vision (Krizhevsky et al., 2012) and the availability of cheap computational hardware has led us to look for a way to automatically detect and classify a disease just by having a computer or a mobile phone process a picture of an affected plant. Given the widespread availability of mobile devices, we believe we can help the vine growers further.

Study was developed to create a large enough database of accurately labeled images and write a software application for optimal and rapid diagnosis of the most important pathogens of vine in our region that would offer additional aid to a vine grower in selecting the best treatment for a diagnosed disease, using the latest machine learning methods of processing and classification of images. 


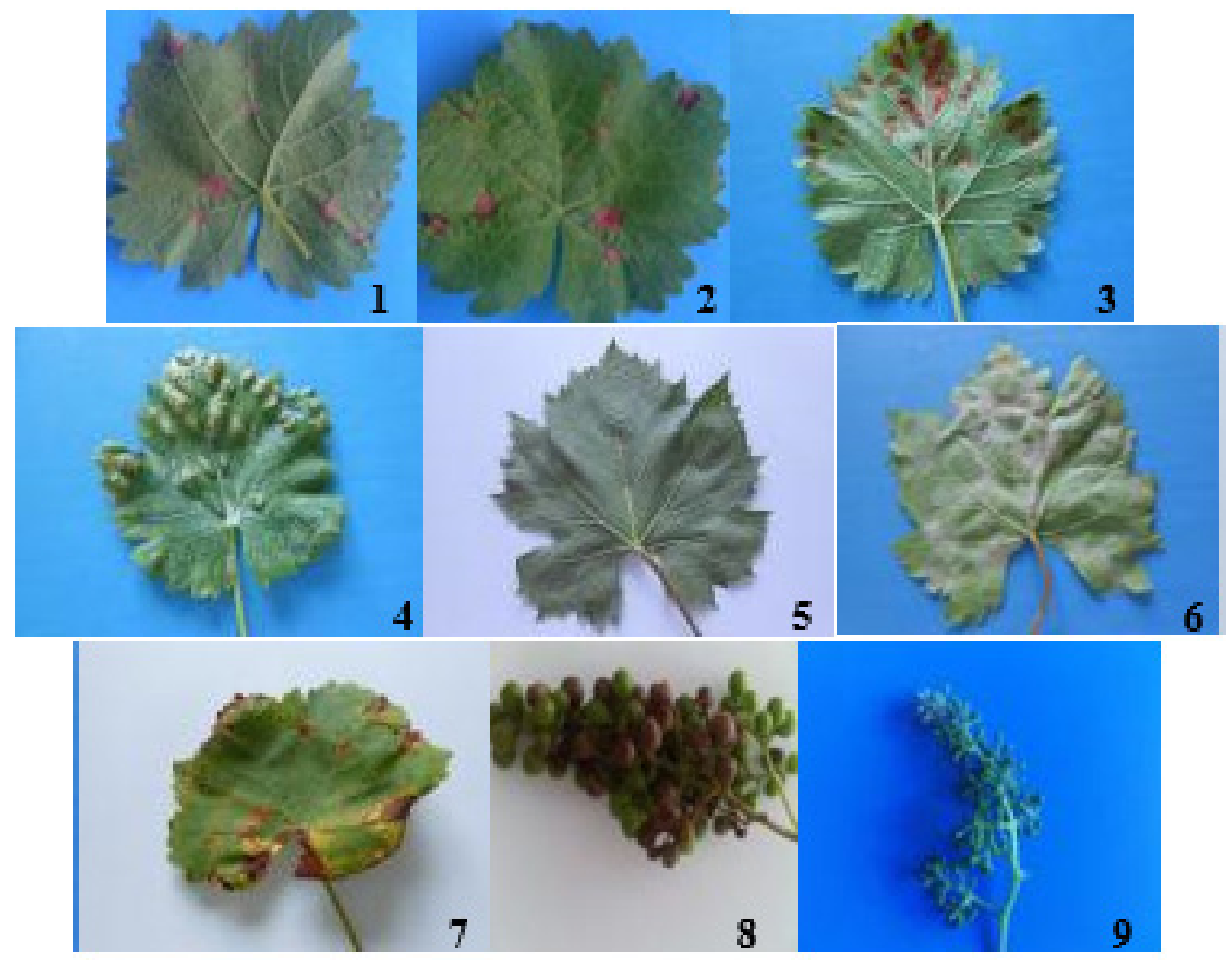

Fig. 1. Examples of leaf healthy and diseased plant leaves and fruits. From left to right: 1) Guignardia bidwelli, back of leaf; 2) Guignardia bidwelli, front of leaf; 3) Colomerus vitis, back; 4) Colomerus, front; 5) Healthy leaf, back; 6) Healthy leaf, front; 7) Plasmopara viticola, back; 8) Plasmopara viticola, front; 9) Plasmopara viticola on fruit; 10) Plasmopara viticola on fruit;

\section{MATERIALS AND METHODS}

The software application was written for iOS, one of the most used mobile operating systems right now. An Android version will also be available soon. It was wanted to write the application natively for performance reasons and to be able to use it offline, without the requirement of an internet connection.

The core of the application is the GoogLeNet convolutional neural network fine tuned to recognize the mentioned vine diseases. It was also experimented with our own model, however, training a convolutional neural network with many layers from scratch requires a lot of computational power but most of all it requires a large database of labeled images in order to obtain a reasonable accuracy and prevent overfitting (Szegedy et al., 2015). Given this and the fact that our database of labeled images was very small, was decided it's best to go with the transfer learning approach where we take an already trained network on a large dataset such as ImageNet and fine tune it.
Transfer learning is desired and has been proven to offer very good results in image classification tasks given a relatively small number of labeled images (Razavian et al., 2014).

Some of the best neural networks architectures today are presented at the Large Scale Visual Recognition Challenge (ILSVRC). It was picked an already trained GoogLeNet model on the ImageNet dataset and to fine tuned this model to recognize vine diseases.

\section{RESULTS AND DISCUSSION}

Our database contains 3000 a labeled image of vine leaves and grapes showing symptoms caused by the most prevalent pathogens in our region: Plasmopara viticola, Uncinula necator, Guignardia bidwelli, Colomerus vitis and Calepitrimerus vitis.

The images were taken in experimental fields at SCDVV Blaj by specialists in phytosanitary protection of vine and were processed by computer scientists. 

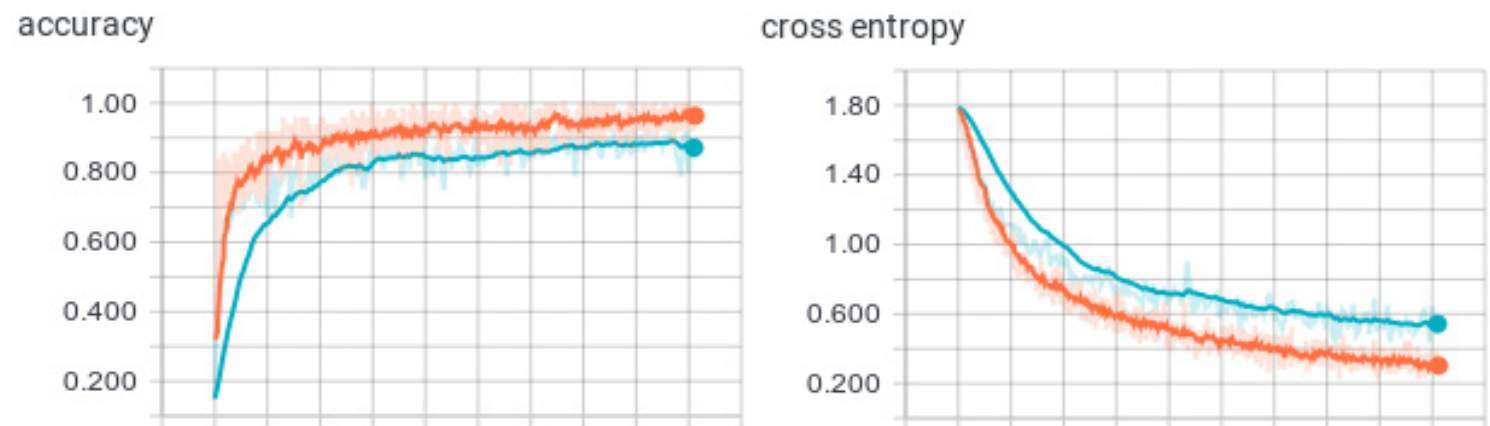

Fig. 2. Fine tuning accuracy and cross entropy. Orange means training and blue means testing.

Tab. 1. Detailed accuracy per class including number of images available per leaf, front and back and per grape

\begin{tabular}{ccccc}
\hline Disease name & No. of images front & No. of images back & $\begin{array}{c}\text { No. of grape } \\
\text { images }\end{array}$ & Accuracy \\
\hline Plasmopara viticola & 417 & 398 & 312 & $94.61 \%$ \\
\hline Guignardia bidwelli & 405 & 405 & 247 & $94.42 \%$ \\
\hline Uncinula necator & 42 & 42 & 0 & $85.17 \%$ \\
\hline Calepitrimerus vitis & 57 & 50 & 0 & $83.13 \%$ \\
\hline Colomerus vitis & 206 & 202 & 72 & $93.23 \%$ \\
\hline
\end{tabular}

Multiple pictures were taken during different hours of a day by rotating each leaf in order to capture more data and allow the model to be more independent of the lighting conditions. One particular note here is the use of a neutral background, either white or blue. The use of a background is of high importance because it eliminates any background noise and increases the accuracy by a noticeable margin.

It was attribute this to the low number of images we gathered per class. The white background was used initially, however a blue background is desirable because some diseases also appear as white patches on a leaf. Different diseases show different signs on the front and the back of a leaf and as such the images were also taken and grouped by either the back or the front of a leaf as shown in Fig.1.

Different mobile phones were used to take the pictures instead of a professional camera in order to get a closer representation of what the model will actually encounter in the field. Because of the low number of images, we had to artificially increase the database size by performing various transformations like rotation, translation, rescaling, flipping and stretching before resizing the images to the accepted model input size.

Then the last layer was reclassified of the already trained GoogLeNet CNN to output softmax scores using stochastic gradient descent (SGD) with a learning rate of $0.002[5,6,7,8,9]$. As shown in Fig. 2, below, the model converges pretty fast.

It was trained different models by trying to find the best results, constantly checking if the model over fit or not. It was noticed that having the test dataset below $10 \%$ produces better results and it's only natural since the model can train on more images. It was achieved an overall top- 1 validation error rate of $9.8 \%$ and a test error rate of $10.7 \%$ after fine tuning the model. Authors believe that better results can be achieved by training not only the last layer of the model but also the layers above it. However, the best way to increase the overall accuracy remains to increase the number of images per class. Was tried with our own model, but we only manage to obtain an accuracy of $82.7 \%$ so far. It is noted that for Calepitrimerus vitis and for Uncinula necator the number of available images was below 50, thus leading to an overall lower accuracy. Was unable to find enough plants 
infected with the two pathogens during the period the pictures were taken.

\section{CONCLUSION}

The software identifies pathogens of vine from images with a reasonable detection rate. The results obtained so far are promising. We believe that by increasing our images database, we will be able to reach a very high level of accuracy.

More work needs to be done in order to obtain a large quantity of expert labeled vine leaf images to increase the detection accuracy in the field where we encounter different conditions and where the leaf background and light varies greatly and currently leads to a low detection rate.

The current obtained results show promise in enabling a quick and efficient resolution of problems caused by the pathogens of vine and the images serve as a database for creating an intelligent module based on machine learning algorithms.

\section{REFERENCES}

1. Ghahramani Z (2015). Probabilistic machine learning and artificial intelligence. Nature 521:452-459.

2. Krizhevsky A, Sutskever I, Hinton GE (2012). Imagenet classification with deep convolutional neural networks in Advances in neural information processing systems. p. 1097-1105.

3. Ali SR, Hossein A, Josephine S, Stefan C (2014). CNN Features off-the-shelf: an Astounding Baseline for Recognition.

4. Szegedy C, Liu W, Jia Y, Sermanet P, Reed S, Anguelov D, Erhan D, Vanhoucke V, Rabinovich A, (2015). Going Deeper with Convolutions, Google Inc. University of North Carolina, Chapel Hill University of Michigan, Ann Arbor 4 Magic Leap Inc.

5. ${ }^{* * *}$ https://en.wikipedia.org/wiki/Overfitting

6. *** http://cs231n.github.io/transfer-learning/

7. ${ }^{* * *}$ http://image-net.org/challenges

8. *** https://en.wikipedia.org/wiki/Softmax_function

9. *** https://en.wikipedia.org/wiki/Stochastic_gradient_ descent

10. ${ }^{* * *}$ https://www.scvblaj.ro 